\title{
X-ray backscatter radiography with lower open fraction coded masks
}

\author{
André A. M. Muñoz ${ }^{\mathrm{a}}$, Anna Vella ${ }^{\mathrm{a}}$, Matthew J. F. Healy ${ }^{\mathrm{a}}$, David W. Lane ${ }^{\mathrm{a}}$, Ian Jupp ${ }^{\mathrm{b}}$, and \\ David Lockley ${ }^{\mathrm{b}}$ \\ ${ }^{a}$ Cranfield Forensic Institute, Cranfield University, Defence Academy of the United \\ Kingdom, Shrivenham, SN6 8LA, United Kingdom \\ ${ }^{\mathrm{b}}$ Counter Terrorism \& Security Division, Defence Science and Technology Laboratory, Fort \\ Halstead, TN14 7BP, United Kingdom
}

\begin{abstract}
Single sided radiographic imaging would find great utility for medical, aerospace and security applications. While coded apertures can be used to form such an image from backscattered X-rays they suffer from near field limitations that introduce noise. Several theoretical studies have indicated that for an extended source the images signal to noise ratio may be optimised by using a low open fraction $(<0.5)$ mask. However, few experimental results have been published for such low open fraction patterns and details of their formulation are often unavailable or are ambiguous. In this paper we address this process for two types of low open fraction mask, the dilute URA and the Singer set array. For the dilute URA the procedure for producing multiple 2D array patterns from given 1D binary sequences (Barker codes) is explained. Their point spread functions are calculated and their imaging properties are critically reviewed. These results are then compared to those from the Singer set and experimental exposures are presented for both type of pattern; their prospects for near field imaging are discussed.
\end{abstract}

Keywords: Coded masks, coded apertures, lower open fraction, 3D printed, X-ray backscatter, dilute URA, singer set.

\section{INTRODUCTION}

The concept of coded aperture or coded mask (CM) imaging dates back to the 1960s, and has been applied to X-ray and $\gamma$-ray astronomy. ${ }^{1,2}$ A variant of CMs called the Uniformly Redundant Array ${ }^{3}$ (URA) was introduced in the 1970s, containing an autocorrelation function (ACF) with perfectly flat side-lobes. The URA is commonly used in CM imaging due to it's superior imaging properties, when compared to others.

The open fraction or mask/aperutre $\operatorname{density}^{4}(\rho)$ is the fraction of open elements within a mask array. Investigation into the relationship between the open fraction and a mask's imaging properties can be traced back as far as the 1970s, with Gunson \& Polychronopulos ${ }^{5}$ who proposed that lower open fraction masks (i.e, $<0.5)$ are optimally suited for imaging lower background scenes within the context of far-field astronomy. The URA has a limited open fraction of 0.5 , which is suited for imaging very low intensity sources ${ }^{6,7}$ such as faint stars. Simulations of SAX X-ray satellite wide field camera (SAX-WFC) ${ }^{7}$ observations of point sources for three sets of data (galactic centre field, large magellanic cloud field and Crab nebular field) revealed higher signal to noise ratio (SNR) on average at lower open fractions between $0.25-0.33$, and also indicate that the SNR was higher imaging faint sources with lower open fraction masks than those with higher open fraction. However, when considering near-field backscattered X-rays, there is limited published research on imaging with low open fraction coded masks. Nevertheless, research has been conducted for applications in the medical industry by simulating images from coded masks of various open fractions. Simulated images from the Modified Uniformly Redundant Array ${ }^{8}$ (MURA) were compared to low open fraction masks. This

Further author information: (Send correspondence to André Muñoz)

André Muñoz: E-mail: a.munoz@cranfield.ac.uk 
includes a No Two Holes Touching mask ${ }^{9}$ (NTHT) array of a MURA pattern with an open fraction of 0.125 and a 'New System' ${ }^{\prime 10}$ array with an open fraction 0.097. The results revealed that the 0.5 open fraction MURA had the best SNR, shortly followed by the NTHT of the MURA pattern, and the 'New System' array ranking significantly lower. ${ }^{11}$ Ideally, a URA with lower open fraction would be the optimum mask for further research due to the shape of its ACF, but true low open fraction URAs are not known to exist thus far. ${ }^{4,7,11,12}$ In addition, the number of lower open fraction arrays with flexibility in its vector sizes are limited, except the random array. ${ }^{12}$

The overall aim of this paper, is to investigate the imaging properties of lower open fraction CMs for use with near-field backscattered X-rays. This is achieved by systematically obtaining exposures of a radioactive ${ }^{241} \mathrm{Am}$ source and an extended scene; using different types of coded masks. In addition, the mathematical construction technique of each coded masks array is presented along with an example of the mask pattern. Overall, this work could potentially enable a staring array backscatter X-ray concept to be realised with greater flux or faster measuring times, when compared to a pinhole aperture imaging system.

\section{CODED MASK ARRAYS}

Four coded mask patterns were chosen based on their open fraction and their predicted imaging abilities. A 19 MURA was selected as a standard for comparison due to its perfect autocorrelation properties, open fraction of 0.5 and wide vector sizes. The second mask selected was a 13 dilute URA, ${ }^{13}$ which will be termed DURA for the remainder of this paper. The DURA has near prefect autocorrelation properties, with a variety of open fractions less than 0.5. Also, a $17 \times 21$ Singer set mask pattern was chosen, as this pattern has been described to obtain the closest optimal SNR for all low open fraction masks. ${ }^{14}$ The final selected mask for comparison was a 19 random array, which will be refereed to as RANDA for the remainder of this paper. This was added to the list of comparison due to its flexibility with its vector size and range of open fractions. All masks mentioned above were 3D printed and cast with a tungsten/epoxy mixture, and therefore retained their true open fractions. Furthermore, all coded masks were compared to a lower open fraction version of their original mask pattern constructed from solid tungsten alloy. To avoid confusion between a theoretical and physical CM all theoretical CM patterns in digital form will be termed array patterns, whilst, physically constructed CMs are refereed to as mask patterns.

Construction methods of 2D array patterns are set out below for the MURA, DURA, Singer set and RANDA. An introduction into the mask properties are given along with calculating the point spread functioin (PSF) of each array pattern (see Fig.1). The PSF in this case was calculated using the fast normalized cross correlation function (see E.q.1) in MATLAB ${ }^{\circledR},{ }^{15,16}$ where $R$ is the reconstructed image (in this case it is the PSF), $D$ representing the encoded image or array (in this case this is the mask array $A$ ), $G$ is the decoding array, and $\bar{D}$ and $\bar{G}$ is the mean of $D$ and $G$.

$$
R(u, v)=\frac{\sum_{x, y}\left[G(x, y)-\bar{G}_{u, v}\right][D(x-u, y-v)-\bar{D}]}{\sqrt{\sum_{x, y}\left[G(x, y)-\bar{G}_{u, v}\right]^{2} \sum_{x, y}[D(x-u, y-v)-\bar{D}]^{2}}}
$$

Each array or mask pattern $A$ with open and closed elements of 1 and 0 was cross correlated with the decoding array pattern $G$ using the balance correlation method, ${ }^{6}$ which follows the conditions in Eq.2. ${ }^{8}$

$$
G_{i, j}=\left\{\begin{array}{cc}
1 & \text { if } A_{i, j}=1 \\
\eta & \text { if } A_{i, j}=0
\end{array}\right\} \quad \text { where, } \eta=-\frac{\rho}{1-\rho}
$$


However, the MURA's decoding array was subject to slight modification as stated by Fenimore \& Gottesman, ${ }^{8}$ which follows the condition in Eq.3

$$
G_{1,1}=1, \quad \text { if } A_{1,1}=0
$$

\subsection{Modified Uniformly Redundant Arrays}

MURAs are a direct extension of the URA family. Unlike twin prime (TP) URAs with vectors $q$ and $p$ adhering to $q-p=2$, MURAs follow the rule $q-p=0$. Consequently, MURAs are perfectly square arrays whose vectors can be any prime number. As a result, this increases the option of array sizes. MURAs are created from the same binary sequence as TP URAs that share an open fraction of 0.5 and posses similar ACF or PSF properties (see Fig.1). Centred MURAs can be symmetrical or non-symmetrical based on its vector length $(L)$ (see Eq.4), where $L$ is a prime number.

$$
L=\left\{\begin{array}{ll}
4 m+1 & \text { for symmetric } \\
4 m+3 & \text { for non-symmetric }
\end{array}\right\} \quad \text { where, } m=1,2,3 \ldots
$$

The $1 \mathrm{D}$ binary sequence $A=\left[A_{i}\right]_{0}^{L-1}$ is then generated using the conditions in Eq.5, where modular arithmetic determines each element in the sequence by placing replacing 1 s where each element is a quadratic residue of modulo $L$.

$$
A_{i}= \begin{cases}0 & \text { if } i=0 \\ 1 & \text { if } i \text { is a quadratic residue modulo } L \\ 0 & \text { otherwise }\end{cases}
$$

An inverse 1D binary sequence $A^{\prime}$ of $A$ is then produced so that each 0 element becomes 1 and all original elements with a value of 1 are changed to 0 (see Eq.6, and 7 for an example of an inverse 5 MURA 1D binary sequence).

$$
\begin{aligned}
& A_{i}=11001 \\
& A_{i}^{\prime}=00110
\end{aligned}
$$

When creating a 2D array pattern, MURA vectors $i$ and $j$ both share the same $1 \mathrm{D}$ binary sequence $A$. That is to say $A_{i}=A_{j}$ and the final mask array template was mapped so that all rows starting with 1 were replaced with $A_{i}$ and rows beginning with 0 were replaced with $A_{i}^{\prime}$ (see example in Fig.2a-c). Subsequently, $A_{i, 1}=1$ then $A_{1, j}=0$ (as demonstrated in Fig.2d) completes the final mask array. Furthermore, the mask array was centred by right shifting and down shifting the rows and vectors of the array by half its vector length, which was then rounded down to the nearest integer (see Fig.2e for an example).

\subsection{Dilute Uniformly Redundant Arrays}

The dilute URA or DURA is a low open fraction array that is derived from known Barker codes. ${ }^{13}$ The DURA contains characteristics of URAs being uniformly redundant with a flat plateau for its PSF. However, DURAs are low open fraction arrays that has similar features in its PSF to the Non Redundant Array (NRA) ${ }^{17}$ (see Fig.1 for PSF). The NRA's PSF possesses a flat plateau with non flat side-lobes that are uniform and then oscillate beyond some distance. ${ }^{13,18,19}$ DURAs contain non flat side-lobes of its PSF which can be removed according to Wild. ${ }^{13}$ For the remainder of this paper the term 'background noise' will refer to a non flat, non uniform plateau and side-lobes of a PSF. This is after all, unwanted signal.

Publications on dilute URAs, 2D example of arrays and their construction technique are rare. 1D binary sequences for dilute URAs have been published with their lengths $L$ and are described in Eq.8, where $K$ is 

(a) MURA
(b) DURA
(c) RANDA
(d) Singer Set
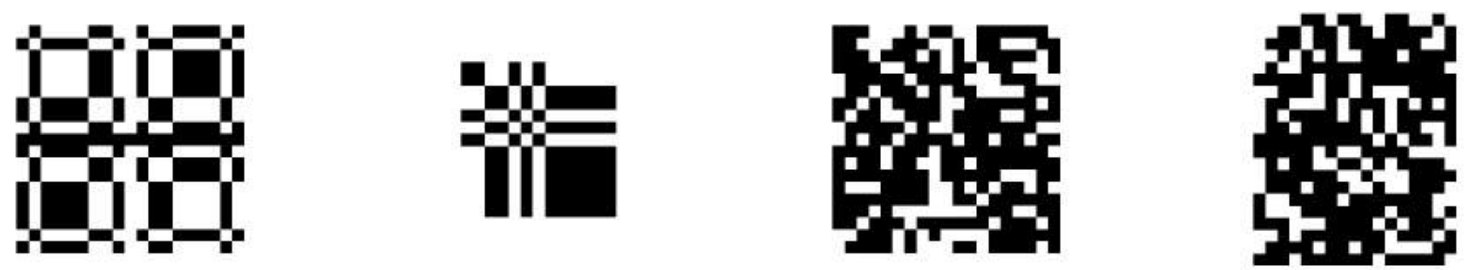

3D PLOT OF THE PSF FOR EACH ARRAY PATTERN

(e)
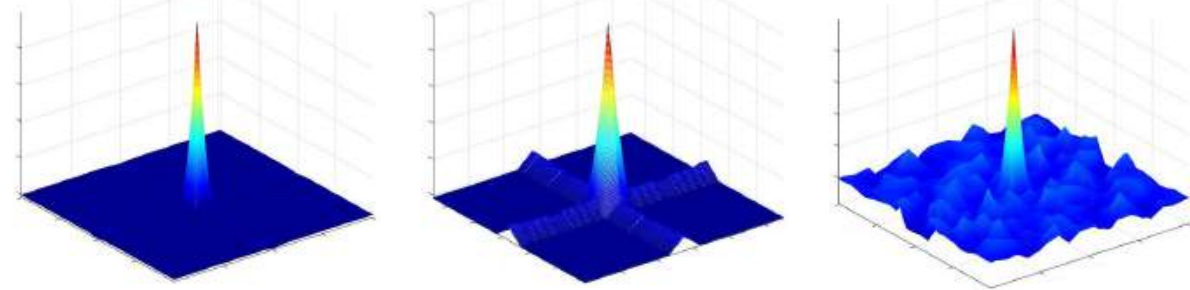

(g)

(h)

\section{NTHT VERSION OF THE ARRAY PATTERN}

(i) MURA NTHT

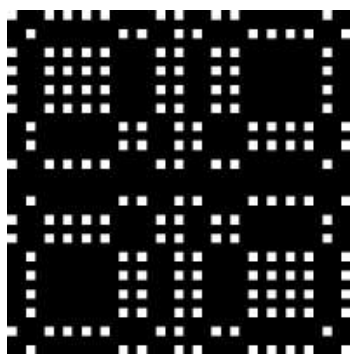

(j) DURA NTHT

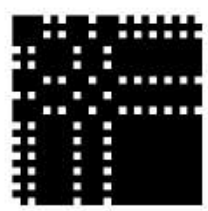

(k) RANDA NTHT

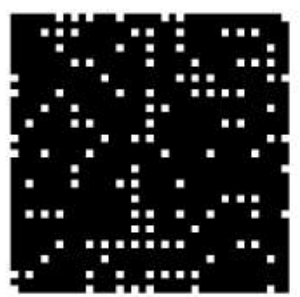

(l) Singer Set NTHT

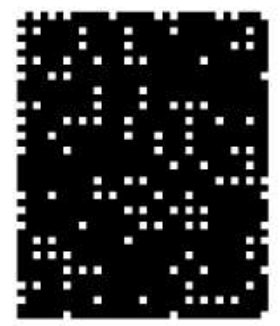

3D PLOT OF THE PSF FOR EACH NTHT VERSION OF THE ARRAY PATTERN

$(\mathrm{m})$

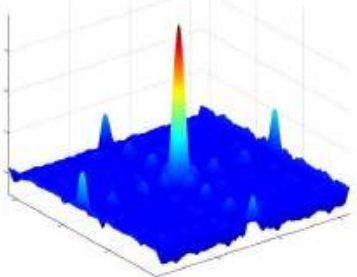

(n)

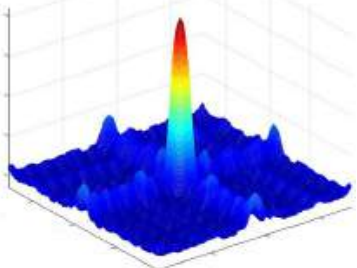

(o)

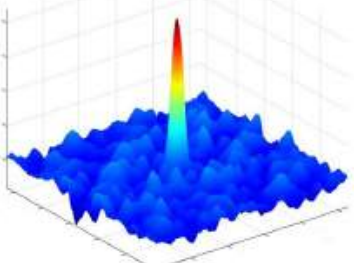

(p)

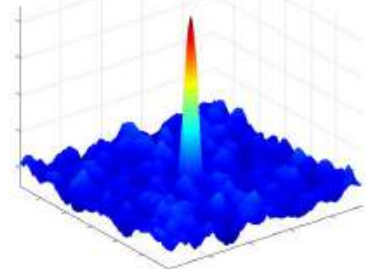

Figure 1: The calculated PSF of each coded mask. 
(a)

(b)

(c)

(d)

(e)

Figure 2: Figure 2a - c demonstrates the mapping process. Subsequently, the initial column and row was replaced with $1 \mathrm{~s}$ and 0 s respectively in Figure $2 \mathrm{~d}$ to complete the mask array. Element $A_{1,1}$ was bold faced in figure $2 \mathrm{~d}$ to show where the MURA decoding array modification occurred (see Eq.3). Finally, Figure 2e shows a centred MURA array after right shifting and down shifting each row and vector of the array.

the number of open elements. Known 1D binary sequences or codes exist for lengths 13,21,31,57 and 73, with multiple binary options for some lengths.

$$
L=K(K-1)+1
$$

The adopted construction technique of $2 \mathrm{D}$ dilute URAs involved a mapping method similar to how the MURA was created (see example in Fig.2a - c). Although the open fraction of a dilute URA's 1D binary sequence is low, using the mapping method with the original code would result in arrays with high open fractions $>0.5$. To obtain lower open fractions $<0.5$, the initial $1 \mathrm{D}$ binary sequence was an inverse of the original, as in Eq.6, and 7. Unlike the MURA, where the mapping process starts with the original 1D binary sequence $A_{i}$ in Fig.2a, this began with the inverse 1D sequence for a 2D DURA and the original sequence followed as in Fig.2b. The 2D DURA mask array was then complete after the process similar to that in Fig.2c.

\subsection{Random array}

Random arrays are arrays with randomly spaced open elements. The side-lobes of its PSF contain no recognisable features and hidden by a non flat and non uniform plateau (see Fig.1 for an example PSF). Although the random array contain inherent noise (i.e, background noise), it is still possible to extract images without significant loss of quality. However, random array images will always have some degree of quality loss due to inherent noise. Nonetheless, random arrays exceed in potential in other areas such as flexible open fraction. The random array mask used in the experiments below were created in MATLAB ${ }^{\complement}$ based upon the desired open fraction.

\subsection{Singer set}

Singer set arrays are derived from cyclic different sets which have low open fraction and are predicted to have optimum SNR. ${ }^{14,20}$ Similar to the random array, singer sets have flexibility in its open fraction, however, containing less background noise (see Fig.1 for an example PSF). A computer program 'cdsgen.exe'14 was used to generate a pseudo-random sequence $M_{0}$ (see Eq.9) as an output of a feedback shift register using $1 / q$, where $q$ is the open fraction and $N$ is the number of shifts.

$$
M_{0}=\frac{q^{N}-1}{q-1}
$$

Singer set arrays are constructed from vectors $m$ and $n$ as described in Eq.10, where $p$ is the pad value of zeros or subtraction of elements to ensure $m$ and $n$ are co-prime. Background noise within the PSF is largely 
independent of the amount of padding and always less than that of a random array. ${ }^{14}$ This step is critical for the folding process which will be described later.

$$
m \times n=\left\{\begin{array}{ll}
M_{0} & \text { if } m \mid M_{0} \\
M_{0}+p & \text { if } m \nmid M_{0}
\end{array}\right\} \quad \text { where, } p \text { is the } \pm \text { pad value. }
$$

All element values $>0$ in $M_{0}$ were set to 0 while the original zero values were changed to 1 . This process created a binary array of $1 \mathrm{~s}$ and $0 \mathrm{~s}$ for both open and closed elements. The sequence $M_{0}$ is then folded according to the vector size similar to the example $M$ in in Fig.3.

$$
M=1, \quad 2, \quad 3, \quad 4, \quad 5, \quad 6, \quad 7, \quad 8, \quad 9, \quad 10, \quad M=\left|\begin{array}{ll}
1 & 6 \\
2 & 7 \\
3 & 8 \\
4 & 9 \\
5 & 10
\end{array}\right|
$$

Figure 3: (Left) The above shows an example of a 1D sequence $M$ folded into a (right) 2D array. Note, the above is just for demonstration purposes and in reality, the $2 \mathrm{D}$ array would be co-prime with binary elements.

\subsection{No Two Holes Touching}

All of the masks mentioned above naturally incorporate a structure that is not self-supporting. As a result, constructing a physical mask can be difficult. According to Fenimore and Cannon, ${ }^{9}$ NTHTs are versions of a mask array with blank or closed elements placed in between each row and column of the original mask array so that no two holes or open elements are ever touching. The addition of these closed elements reduce the open fraction of the original mask which can be described in Eq.11 for NTHTs with circular apertures. $\phi_{N}$ is the number of mask elements, $r$ is the radius of the apertures and $\mathrm{CM}_{A}$ is the mask area. NTHTs have the advantage of supporting the structure of a mask, however, at the expense of introducing background noise into the final image (see Fig.1 for examples of a PSF).

$$
\rho_{N T H T}=\frac{\phi_{N} \pi r^{2}}{\mathrm{CM}_{A}}
$$

\section{EXPERIMENTAL SETUP}

To evaluate the CMs, experiments were done using an ${ }^{241} \mathrm{Am}$ radioactive source to determine the PSF and X-ray backscatter off two test objects using a $800 \mathrm{~W} \mathrm{VJT}^{\circledR}$ X-ray source ${ }^{21}$ operating at $100 \mathrm{kV}, 5 \mathrm{~mA}$. An X-ray sensitive Photonic Science ${ }^{\circledR}$ iCCD camera $^{22}$ was used to detect backscattered X-rays with a square active region of $80 \times 80 \mathrm{~mm}$ (see Fig. 4 for setup). Both objects and radioactive source were centred within the detector's field of view (FOV), with the radioactive source placed at a distance of $1 \mathrm{~m}$ from the mask and $1.5 \mathrm{~m}$ for the backscattered objects. The distance from the mask to the focal plane of the detector was set to $111 \mathrm{~mm}$ for the backscattered objects and $141 \mathrm{~mm}$ for the radioactive source. The test objects were a quadrant of three blocks and a cylinder, and a pair of scissors placed in front of a parrafin wax block (see Fig.5). Two types of masks were used for the experiments; a NTHT Wolfmet HA $190^{23}$ tungsten alloy version of the original mask pattern and a 3D printed polyactic acid (PLA) mould filled with tungsten powder and epoxy $\operatorname{resin}^{24}$ (see Fig.5). All masks were $2 \mathrm{~mm}$ thick for its X-ray attenuating material and mosaicked $2 \times 2$ minus one row and one column to reduce the effects of partially coded field of view (PCFOV) ${ }^{8,19,25,26}$ (see Table 1 for details on the CMs used). When constructing the coded mask, care was taken so that the mask unit pattern was smaller than the detector's active region (as a result this governed the choice of vector sizes for each mask). This would then ensure that the shadow of the CM's unit pattern projected a full cycle within the detector's active region (see Table 1 for detailed mask parameters). It is important to note that 
the open fraction of the MURA NTHT was slightly lower than the other masks. This was because it had been fabricated with a spacing of $3.21 \mathrm{~mm}$ for an earlier set of experiments. Those for the other NTHT masks were made with a slightly smaller spacing of $2.67 \mathrm{~mm}$.

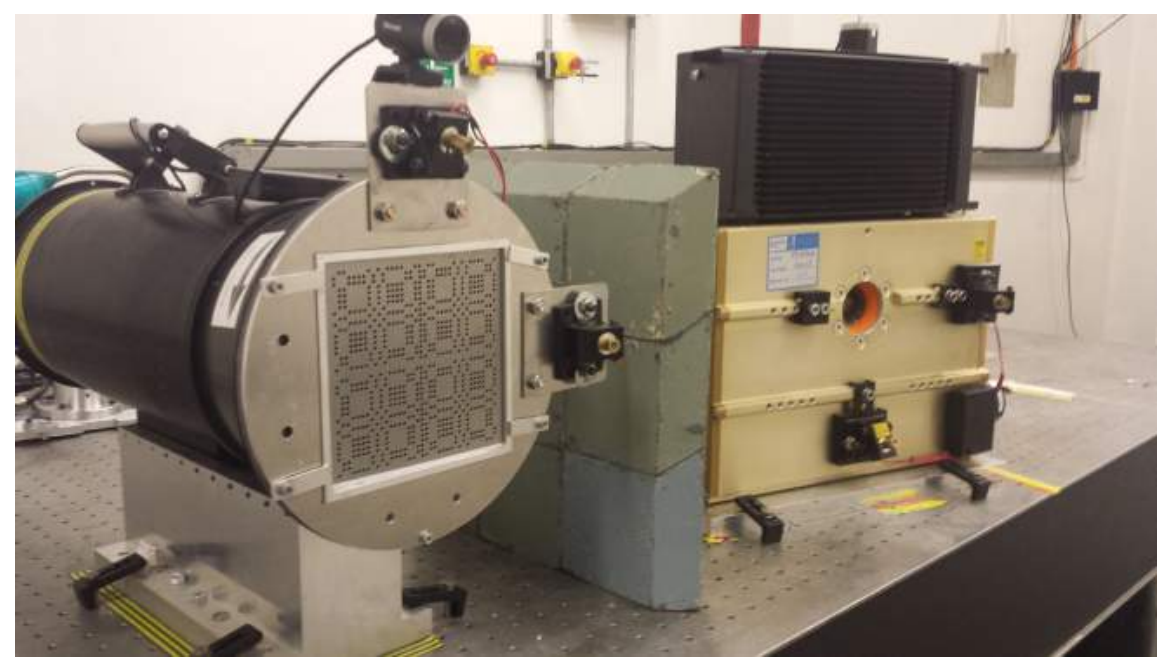

Figure 4: Photonic Science ${ }^{\circledR}$ X-ray camera with a VJT ${ }^{\circledR}$ X-ray source beside it.

Table 1: Parameters for the coded mask used in the experiment below.

\begin{tabular}{|l|c|c|c|c|c|}
\hline Mask type & Vector size & $\begin{array}{c}\text { Unit pattern } \\
(\mathrm{mm})\end{array}$ & $\begin{array}{c}\text { Pattern Size } \\
(\mathrm{mm})\end{array}$ & Open fraction & No. of holes \\
\hline MURA & $19 \times 19$ & $38 \times 38$ & $74 \times 74$ & 0.50 & - \\
DURA & $13 \times 13$ & $26 \times 26$ & $50 \times 50$ & 0.43 & - \\
RANDA & $19 \times 19$ & $38 \times 38$ & $74 \times 74$ & 0.33 & - \\
Singer set & $17 \times 21$ & $34 \times 42$ & $66 \times 82$ & 0.33 & - \\
MURA NTHT & $37 \times 37$ & $60 \times 60$ & $117 \times 117$ & 0.16 & 252 \\
DURA NTHT & $25 \times 25$ & $34 \times 34$ & $66 \times 66$ & 0.18 & 440 \\
RANDA NTHT & $37 \times 37$ & $50 \times 50$ & $98 \times 98$ & 0.14 & 464 \\
Singer set NTHT & $33 \times 41$ & $45 \times 55$ & $87 \times 109$ & 0.15 & \\
\end{tabular}

\subsection{Decoding Process}

The process of encoding an image $D$ can be described in Eq.12, where $O$ is the source or object, $A$ is the coded mask array, $\otimes$ is the correlation operator and $\mathrm{N}$ is the addition of noise. Each encoded array or exposure was cropped in the centre to match only the projected shadow of the masks unit pattern (see Fig.6 for an example). It was found that cropping out the mosaic from $D$ was essential for removing artefacts introduced by the mosaic and correlation process. ${ }^{25}$

$$
D=(O \otimes A)+\mathrm{N}
$$

As the source/object in the experiments were subject to near field magnification $\left(N_{m}\right)$, the projected unit pattern shadow was larger. Therefore, Eq.13 was used to scale $G$ so that the unit pattern from both $D$ and 
(a) Quadrant

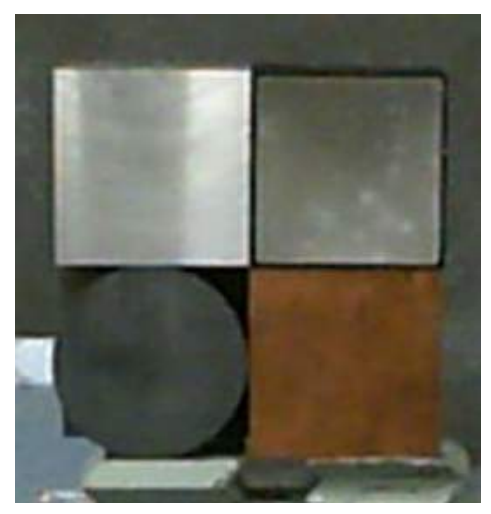

(c) NTHT HA 190

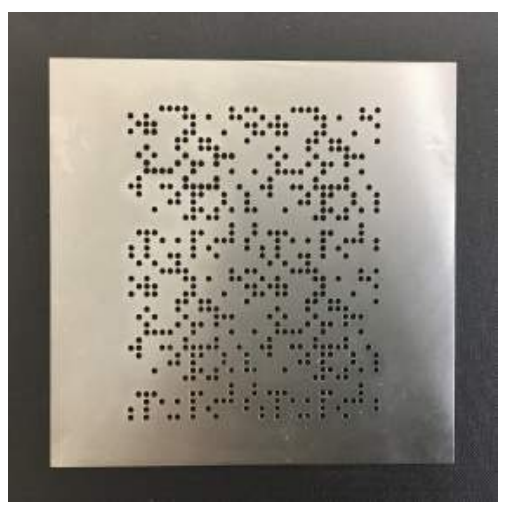

(b) Scissors

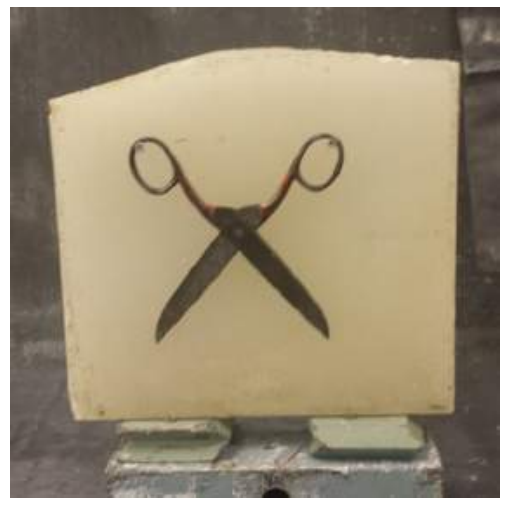

(d) $3 \mathrm{D}$ printed $\mathrm{CM}$

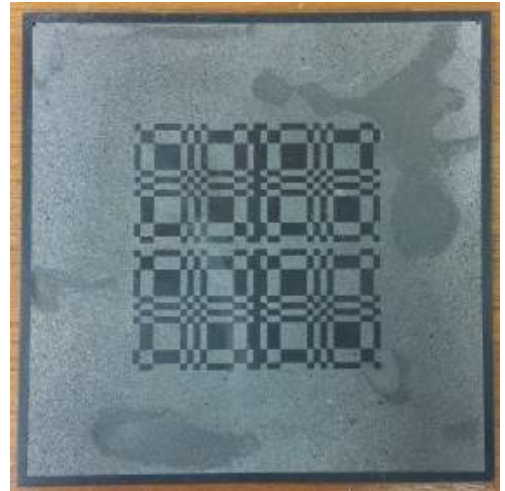

Figure 5: (a) shows a quadrant and (b) pair of scissors in front of a wax block. An example tungsten NTHT and $3 \mathrm{D}$ printed mask are revealed in $(\mathrm{c})$ and $(\mathrm{d})$.

$G$ were the same size, where $a$ is the source/object to mask distance and $b$ is the distance between the mask and the focal plane of the detector.

$$
N_{m}=\frac{a+b}{b}
$$

For cross correlation to occur, either $D$ or $G$ must be larger than the other. $A$ and $G$ share the same array pattern with the exception of conditions in Eq.2 being applied for $G$ 's open and closed elements. Both arrays were mosaicked, thus, were larger than the cropped unit pattern of $D$. With the size compliance of both arrays, final reconstructed images and PSF were correlated using Eq.1 with no further image enhancement (result are presented later on in the paper). Each reconstructed radioactive source image comprised of a single $300 \mathrm{~s}$ exposure with the coded mask in its normal orientation. All reconstructed backscattered images contained two $5 \mathrm{~s}$ exposures; one with the mask in its normal orientation and another with the mask rotated 90 degrees to remove unwanted artefacts that travel with the mask's rotation. ${ }^{27}$ Ultimately, both decoded exposures were summed with a total exposure time of $10 \mathrm{~s}$. The exposures presented in this paper were automatically reconstructed in $\mathrm{MATLAB}^{\circledR}$ with a total processing time of less than $1 \mathrm{~s}$ on a standard laptop computer. The process included all cropping, scaling, cross correlation and summations. 


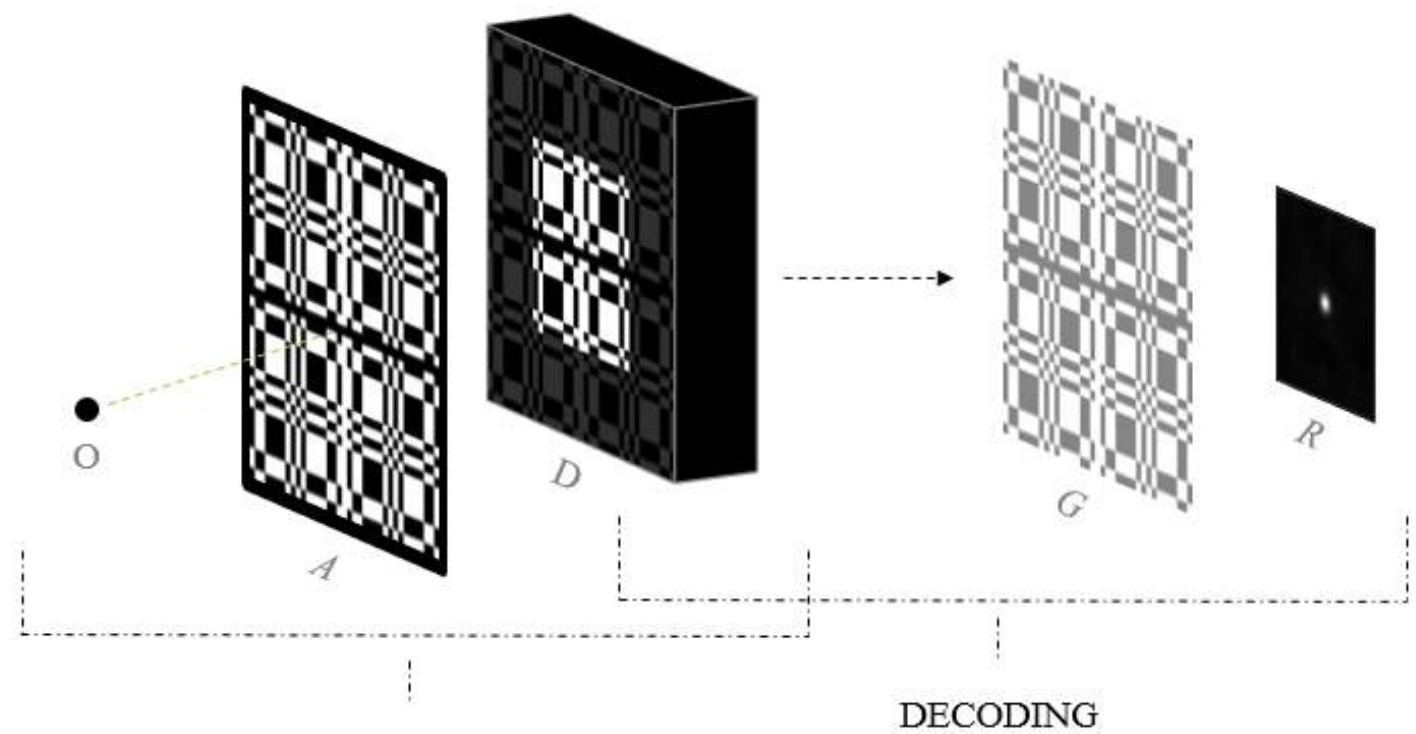

ENCODING

Figure 6: Source or object $O$ and the coded mask $A$. $D$ shows a cropped projection of the coded mask unit pattern onto the detector's active region. $G$ is the decoding array pattern along with the reconstructed image $R$.

\section{RESULTS}

The image resolving abilities were found for each coded mask array by taking a single 1D slice of the central region of the PSF determined from the ${ }^{241} \mathrm{Am}$ exposure, then finding the full width at half maximum (FWHM) between the pixel intensity of 0 and the max value. This took place in both the $\mathrm{X}$ and $\mathrm{Y}$ direction of the array, which was then averaged. In addition, the SNR (see Eq.14) of each mask was found by calculating the mean signal $\left(\mu_{\mathrm{S}}\right)$ of the PSF and the standard deviation of the remaining side lobes and plateau $\left(\sigma_{\mathrm{N}}\right)$ (Table 2). The theoretical PSF for each mask are compared to the images of ${ }^{241} \mathrm{Am}$ in Fig.7 8. Backscattered exposures were also taken of objects in Fig.5a and b and are shown in Fig.9 and 10. The central signal in the radioactive exposures appear broader than its calculated counterpart. This is because the radioactive source was not a true point source and was shaped like a toroid with a size of approximately $2 \mathrm{~cm}$ in diameter.

$$
\mathrm{SNR}=\frac{\mu_{\mathrm{S}}}{\sigma_{\mathrm{N}}}
$$


PSF OF ARRAY PATTERN

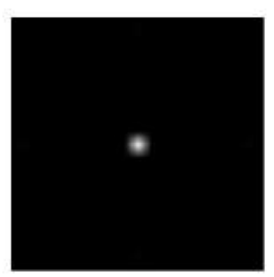

MURA

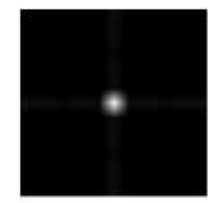

DURA

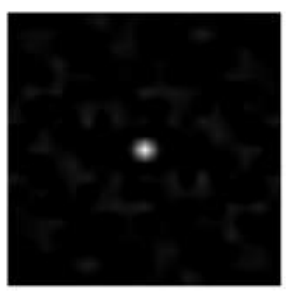

Random array

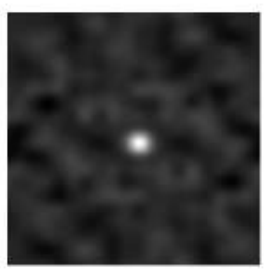

Singer set

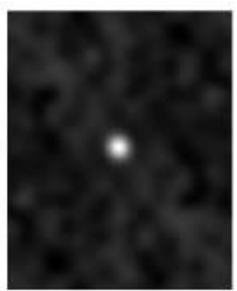

Figure 7: (Left) theoretical PSF of the array pattern. (Right) experimental decoded images of the ${ }^{241} \mathrm{Am}$ radioactive source. 
PSF OF NTHT ARRAY PATTERN

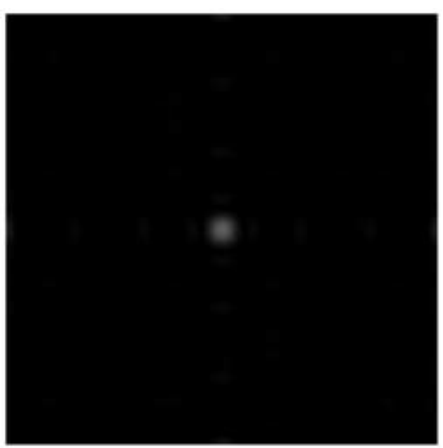

MURA

DURA

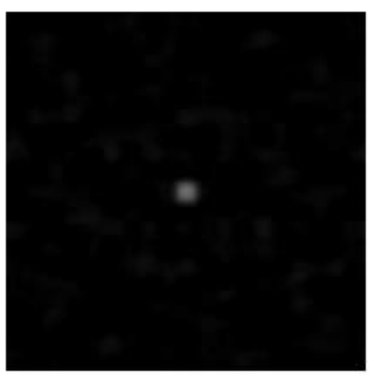

Random array

Singer set

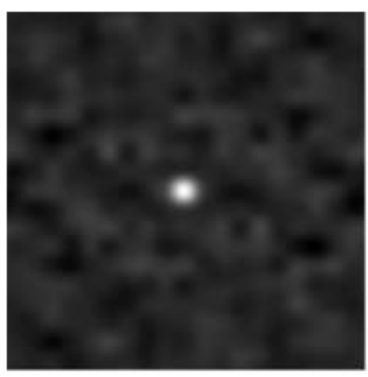

${ }^{241}$ Am EXPOSURES WITH NTHT
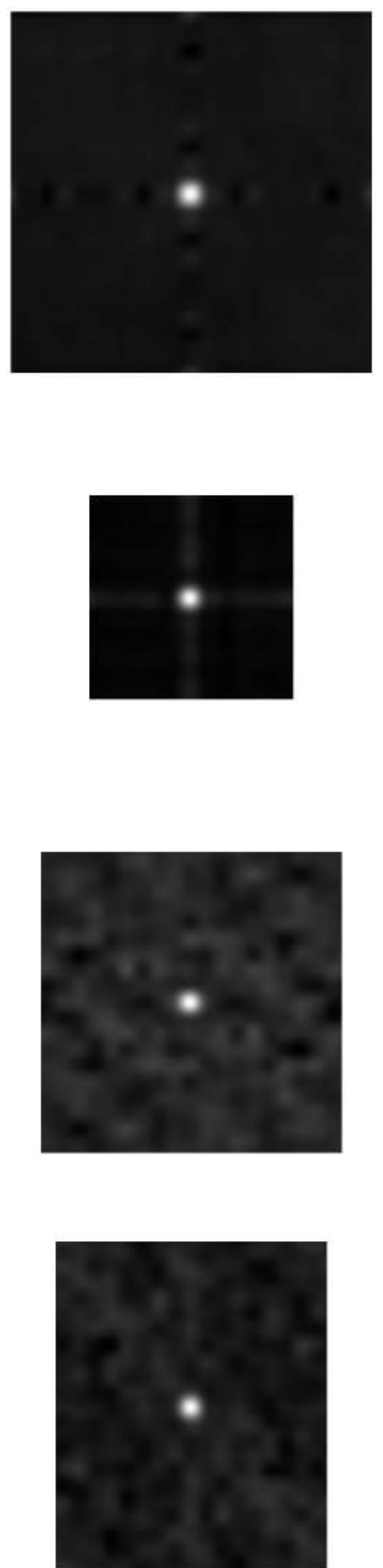

Figure 8: (Left) theoretical PSF of the NTHT array pattern. (Right) experimental decoded images of the ${ }^{241} \mathrm{Am}$ radioactive source. 
3D PRINTED
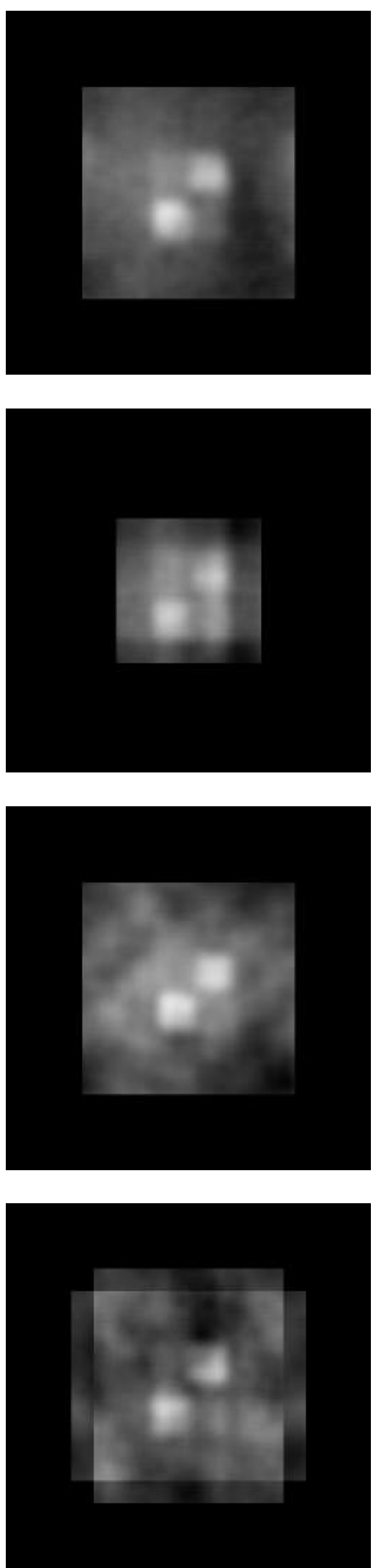

NTHT

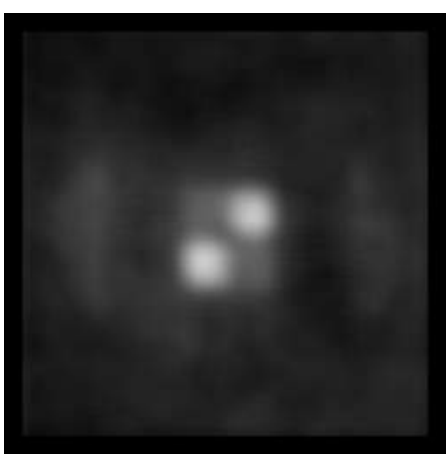

DURA
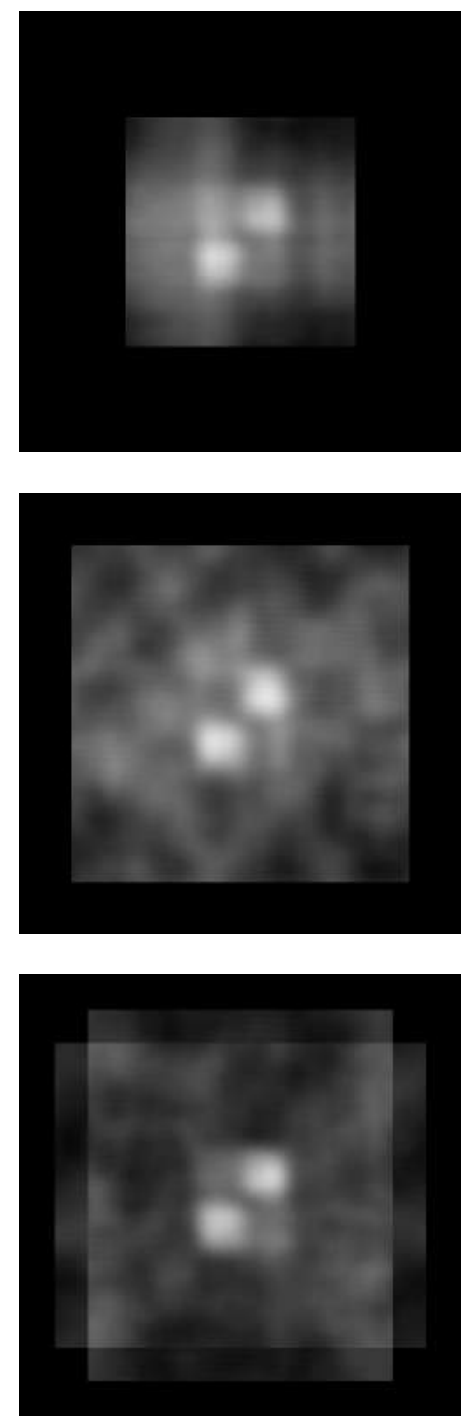

Figure 9: Experimental backscattered X-ray images of the quadrant in Fig.5 was taken with each mask. All images were a sum of $2 \times 5 \mathrm{~s}$ exposures with the coded mask in the correct orientation and then rotated 90 degrees. 
3D PRINTED
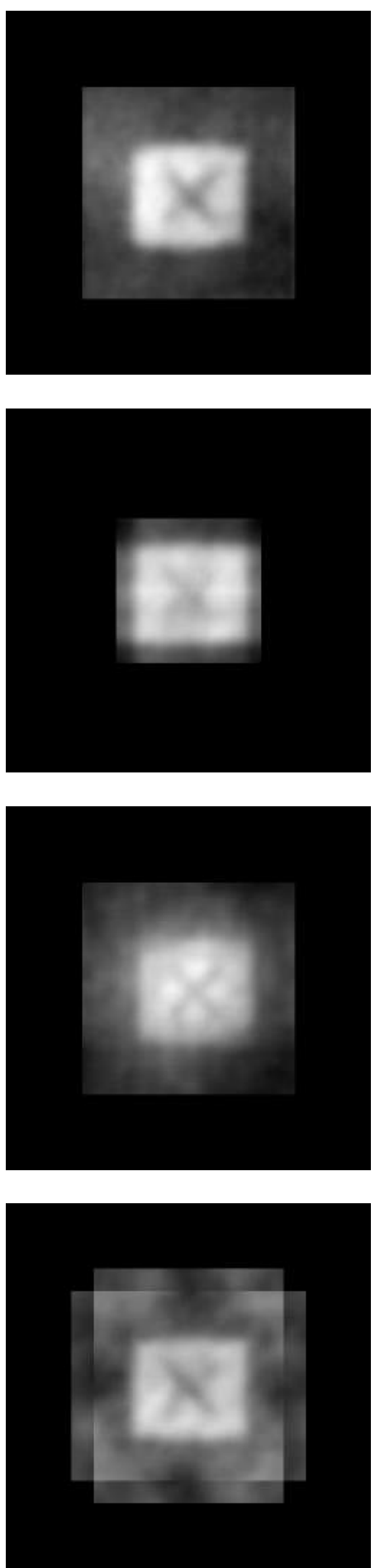

NTHT

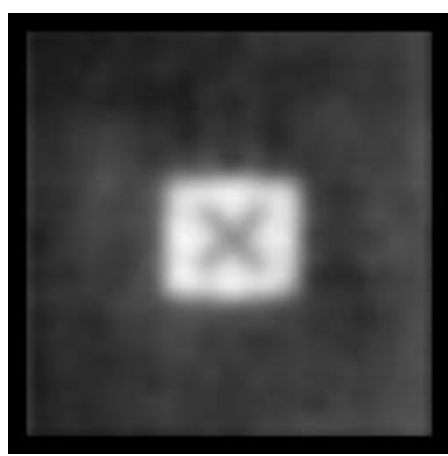

DURA
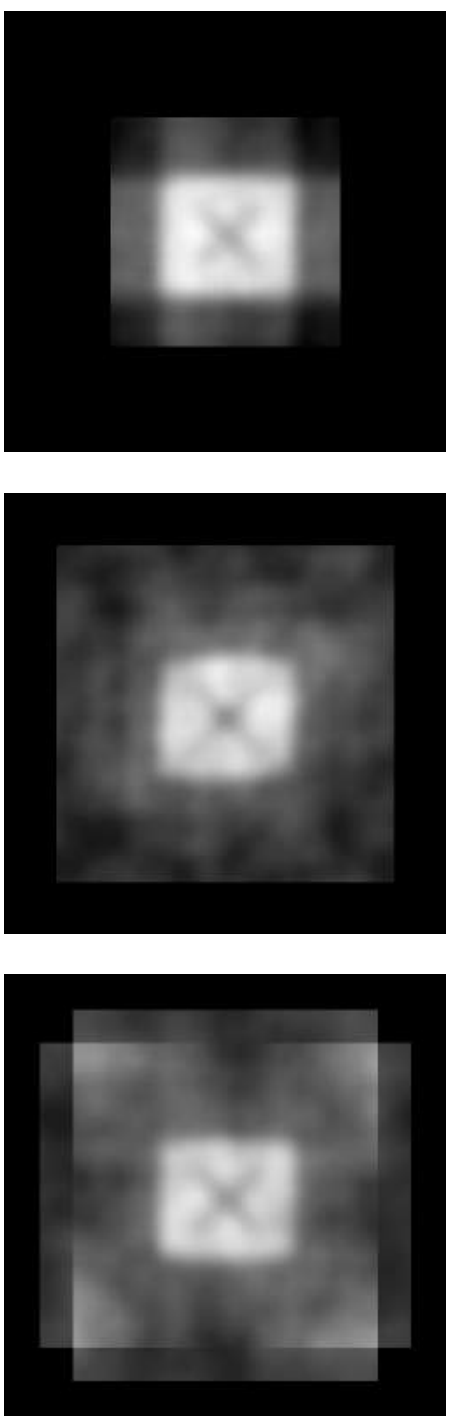

Figure 10: Experimental backscattered X-ray images of a pair of scissors in front of a wax block in Fig.5 was taken with each coded mask. All images were a sum of $2 \times 5 \mathrm{~s}$ exposures with the mask in the correct orientation and then rotated 90 degrees. 
Table 2: FWHM and SNR of the ${ }^{241} \mathrm{Am}$ exposures and PSF for each array and mask pattern.

\begin{tabular}{|c|c|c|c|c|}
\hline MASK ARRAY PATTERNS & $\begin{array}{l}\text { FWHM } \\
\text { (pixels) }\end{array}$ & $\mu_{\mathrm{S}}$ & $\sigma_{\mathrm{N}}$ & SNR \\
\hline \multicolumn{5}{|c|}{ THEORETICAL PSF FOR THE ARRAY PATTERN } \\
\hline $\begin{array}{l}\text { MURA } \\
\text { DURA } \\
\text { Random array } \\
\text { Singer set }\end{array}$ & $\begin{array}{l}21 \\
21 \\
22 \\
21\end{array}$ & $\begin{array}{l}66.850 \\
70.353 \\
71.507 \\
71.594\end{array}$ & $\begin{array}{c}0 \\
3.465 \\
4.849 \\
3.968\end{array}$ & $\begin{array}{c}\mathrm{N} / \mathrm{A} \\
20.304 \\
14.747 \\
18.043\end{array}$ \\
\hline \multicolumn{5}{|c|}{ THEORETICAL PSF FOR THE NTHT ARRAY PATTERN } \\
\hline $\begin{array}{l}\text { MURA } \\
\text { DURA } \\
\text { Random array } \\
\text { Singer set }\end{array}$ & $\begin{array}{l}28 \\
27 \\
28 \\
28\end{array}$ & $\begin{array}{l}34.757 \\
41.964 \\
23.506 \\
42.490\end{array}$ & $\begin{array}{l}1.339 \\
2.595 \\
2.737 \\
2.663\end{array}$ & $\begin{array}{c}25.957 \\
16.171 \\
8.588 \\
15.956\end{array}$ \\
\hline \multicolumn{5}{|c|}{${ }^{241}$ Am EXPOSURES WITH 3D PRINTED MASKS } \\
\hline $\begin{array}{l}\text { MURA } \\
\text { DURA } \\
\text { Random array } \\
\text { Singer set }\end{array}$ & $\begin{array}{l}30 \\
28 \\
28 \\
28\end{array}$ & $\begin{array}{l}119.616 \\
133.062 \\
152.245 \\
135.957\end{array}$ & $\begin{array}{c}3.209 \\
9.995 \\
12.135 \\
10.649\end{array}$ & $\begin{array}{l}37.275 \\
13.313 \\
12.546 \\
12.767\end{array}$ \\
\hline \multicolumn{5}{|c|}{${ }^{241}$ Am EXPOSURES WITH NTHT MASKS } \\
\hline $\begin{array}{l}\text { MURA } \\
\text { DURA } \\
\text { Random array } \\
\text { Singer set }\end{array}$ & $\begin{array}{l}36 \\
30 \\
31 \\
31\end{array}$ & $\begin{array}{l}129.206 \\
132.266 \\
143.406 \\
131.420\end{array}$ & $\begin{array}{c}3.932 \\
7.327 \\
10.432 \\
8.909\end{array}$ & $\begin{array}{l}32.860 \\
18.052 \\
13.747 \\
14.751\end{array}$ \\
\hline
\end{tabular}




\section{DISCUSSION}

The imaging capabilities of a coded mask array can be revealed by calculating its PSF. The optimum PSF for an imaging system would comprise a sharp central peak with uniformly flat side-lobes and plateau. MURAs posses such qualities in a theoretical world, as their PSF contain pure signal (see Fig.1 and Table 2 for examples). The PSF of a DURA is similar to MURA with a uniformly flat plateau. However, it consists of non flat side-lobes that introduce artefacts into the image (see Fig.1, Fig.7 and Table 2 for examples), which is not ideal for imaging. Nevertheless, the DURA has the highest SNR when compared to the other low open fraction masks and it has been theorised that such artefacts can be removed. Consequently, the removal of these artefacts would increase the DURA's status as an ideal imaging mask. The DURA has flexibility in its open fraction which is an advantage over the MURA. When analysing the PSF and imaging properties of the random array, noise is introduced into the image from its non flat, non uniform plateau. The same is true for the Singer set's PSF, however, embodying a slightly smoother plateau than that of the random array's. The slight advantage of the Singer set over the random array is evident in the SNR where the Singer set exceeds that of the random array's. The random array possessed the lowest SNR of all lower open fraction masks above (see Fig.1, Fig.8 and Table 2 for examples).

NTHT versions the original the array pattern are lower in open fraction due to the addition of closed elements. Consequently, NTHTs accommodates a self supporting structure for a fabricated mask, while the SNR, conversely, is reduced. The reason for the reduction in SNR is because NTHTs contain artefacts from its side-lobes of its PSF, with the addition of an non-uniform plateau. Evidently, examples can be seen with the MURA pattern and its NTHT version, where the uniformly flat side lobes and plateau are eliminated in the NTHT array. The SNR for the NTHT versions of the array patterns above rank as follow with the highest SNR first; MURA, DURA, Singer set and the random array having the worst results (see Fig.1, Fig.7 and Table 2 for examples). Calculating SNR involved cropping the central signal of the PSF and ${ }^{241}$ Am source exposure in ImageJ ${ }^{\complement} .^{28}$ However, it must be made clear that there is a degree of uncertainty in the cropping process, as it was performed by eye. This maybe supported by the fact that theoretical SNR values for both NTHTs of the DURA and RANDA were slightly lower than the experimental values, which was not expected.

Exposures were taken of an ${ }^{241} \mathrm{Am}$ source using physically constructed coded masks. A visual comparison of the array pattern PSF and ${ }^{241}$ Am source images revealed similarities. Nevertheless, the addition of noise $\mathrm{N}$ (see Eq.12) from X-ray scatter and photon noise, at the very least, it is clear in the ${ }^{241}$ Am source images. SNR for the ${ }^{241} \mathrm{Am}$ source exposures that were captured with both the 3D printed and NTHT masks confirm results from earlier, with the MURA performing the best, followed by the DURA, Singer set and random array performing last. It must be emphasised that the MURA NTHT mask's hole spacing was slightly larger, which lowers the open fraction and may impact its SNR (see Fig.7, Fig.8 and Table 2). In confirming the results for each mask, backscattered exposures of the quadrant and pair of scissors appear congruous to SNR presented earlier. The signal from objects with the backscattered images appear stronger, with the overall image being less 'washed out' for the MURA exposures. This was then followed by the DURA, Singer set and then the random array (see Fig.9, Fig.10 and Table 2 for examples). It must be noted that, low activity of the source meant it had to be located closer to the camera than the X-ray backscatter object. The X-ray backscatter object was placed further back so that the scene could fit within the imaging systems FOV. A direct comparison with both the object and source having the same distance to the mask and similar aperture to detector distance would have meant performing the whole experiment again. .

In theory, larger array patterns have higher resolving capabilities. Limited by the detector's active region, the chosen vector size of each array was close to 19 elements so that when scaled, a full cycle of the unit array pattern was projected onto the detector. An exception was made for the DURA, as the 13 vector array produced the most desirable imaging properties. In addition, all arrays were scaled so that the smallest element of the array pattern was similar in size, and a comparison of the resolving capabilities of each mask was compared. By measuring the FWHM of each PSF it was found that the results for all array patterns were rather comparable with values varying by \pm 1-2 pixels, except for the MURA NTHT experimental exposures, 
which had a slightly higher FWHM of 5-6 pixel. It is not clear why the results were higher, nonetheless, the effects are almost negligible on this scale.

There was great difficulty in visually comparing the FWHM results to the actual exposures presented earlier, as judging the image resolution by eye may vary one's point of view. Be that as it may, some features of the objects in the backscattered images were not represented entirely correctly. For example, the quadrant shows two square blocks in the upper right and lower left, when the one in the lower left corner should be circular (see Fig.9). The cause of this is not known and was introduced in the reconstruction process.

\section{CONCLUSION}

Overall results from SNR of the theoretical mask and NTHT version, along with the experimental exposures using the 3D printed mask and tungsten NTHT revealed a consistent trend. When analysing the SNR from all four coded mask patterns, images from the 0.5 open fraction MURA outperformed those from all lower open fraction CMs. This was followed by the DURA, Singer set and random array which produces the lowest SNR. Consequently, results from the DURA yielded the best SNR followed by the Singer set when comparing all lower open fraction CMs used in the experiment. This was confirmed for all four experiments comparing the theoretical arrays and their NTHT patterns, along with the physical coded mask and tungsten heavy NTHTs.

The FWHM was measured for each mask's PSF and ${ }^{241} \mathrm{Am}$ source exposures. The results were rather comparable which was expected, due to all smallest element of each array or CM having the same size. However, it was found that the FWHM for the experimental MURA NTHT was slightly higher than all others. The difference in value, nonetheless, was relatively small and can be considered to be negligible.

Overall, in terms of SNR, when analysing the prospect of a MURA, DURA, Singer set and RANDA CM, the MURA possessed the best imaging properties for near-field imaging. For lower open fraction masks the DURA yielded the highest SNR followed by the Singer set. The uniformly flat plateau was a major factor that increased the SNR with the DURA mask. If the non flat side-lobes can be removed as suggested in Wild, ${ }^{13}$ then the DURA would posses imaging qualities equal to that of the MURA.

\section{ACKNOWLEDGEMENTS}

The authors would like to thank Dstl for the financial support for this work.

\section{REFERENCES}

[1] Mertz, L. and Young, N. O., "Fresnel Transformation of Images," in [Optical Instruments and Techniques (Chapman and Hall Ltd)], 305-310 (1961).

[2] Dicke, R. H., "Scatter-Hole Cameras for X-Rays and Gamma Rays," Astrophysical Journal 153, L101 (1968).

[3] Fenimore, E. E., "Coded Aperture Imaging with Uniformly Redundant Arrays," Applied optics 17(3), $337-347$ (1978).

[4] Busboom, A., Elders-Boll, H., and Schotten, H., "Uniformly Redundant Arrays," Kluwer Academic Publishers , 97-123 (1998).

[5] Gunson, J. and Polychronopulos, B., "Optimum Design of A Coded Mask X-Ray Telescope for Rocket Applications," Royal Astronomical Society 177, 485-497 (1976).

[6] Fenimore, E. E., "Coded Aperture Imaging with Uniformly Redundant Arrays.," Applied optics 17(3), 337-347 (1978).

[7] In 't Zand, J., Heise, J., and Jager, R., "The Optimum Open Fraction of Coded Apertures. With an Application of the Wide Field X-ray Cameras of SAX," European Southern Observatory 288, 665-674 (1994). 
[8] Fenimore, E. E. and Gottesman, S., "New Family of Binary Arrays for Coded Aperture Imaging," Applied optics 28(20), 4344-4352 (1989).

[9] Fenimore, E. E. and Cannon, T. M., "Uniformly redundant arrays: digital reconstruction methods.," Applied optics 20(10), 1858-64 (1981).

[10] Jennings, D. and Byard, K., "An extension for residue difference sets," Discrete Mathematics 167168, 405-410 (1997).

[11] Accorsi, R., Gasparini, F., and Lanza, R., "Optimal Coded Aperture Patterns for Improved SNR in Nuclear Medicine Imaging," Elsevier 474, 273-284 (2001).

[12] Busboom, A., Elders-Boll, H., and Schotten, H. D., "Combinatorial design of near-optimum masks for coded aperture imaging," ICASSP, IEEE International Conference on Acoustics, Speech and Signal Processing - Proceedings 4, 2817-2820 (1997).

[13] Wild, W. J., "Dilute Uniformly Redundant Sequences for use in Coded-Aperture Imaging," Optics Letters 8(5), 247-249 (1983).

[14] Shutler, P. M. E., Springham, S. V., and Talebitaher, A., "Mask design and fabrication in coded aperture imaging," Nuclear Instruments and Methods in Physics Research, Section A: Accelerators, Spectrometers, Detectors and Associated Equipment 709, 129-142 (2013).

[15] MathWorks, "MATLAB." Available from: http://uk.mathworks.com/products/matlab/, (Accessed 24 January 2016) (2015).

[16] Lewis, J. P., "Fast Normalized Cross-Correlation," Canadian Image Processing and Pattern Recognition Society 10(11), 120-123 (1995).

[17] Golay, M., "Point Arrays Having Compact, Nonredundant Autocorrelations," Applied optics (61), 272 (1971).

[18] Skinner, G. K., "Imaging With Coded-Aperture Masks," Nuclear Instruments and Methods in Physics Research 221, 33-40 (1984).

[19] Accorsi, R., Design of Near-Field Coded Aperture Cameras for High-Resolution Medical and Industrial Gamma-Ray Imaging, PhD thesis (2001).

[20] Shutler, P. M., Talebitaher, A., and Springham, S. V., "Signal-to-noise ratio in coded aperture imaging," Nuclear Instruments and Methods in Physics Research Section A: Accelerators, Spectrometers, Detectors and Associated Equipment 669, 22-31 (2012).

[21] VJT, "VJ Technology." Available from: http://www.vjt.com/(Accessed 25 June 2017) (2017).

[22] Photonic Science, "Gemstar X-ray Camera." Available from: http://www.photonicscience.co.uk/(Accessed 25 June 2017) (2017).

[23] Wolfmet, "Tungsten Alloys Technical Information." Available from: http://static.mimaterials.com/wolfmet/documents (Accessed 17 November 2016) (2016).

[24] Inc, T. H. P., [Technon / Poly Kit], Available from: http://www.thpap.com/(Accessed 9 February 2016) (2001).

[25] Jupp, I., The Optimisation of Discrete Pixel Coded Aperture Telescopes, PhD thesis, University of Southampton (1996).

[26] Caroli, E., J., S., Di Cocco, G., Natalucci, L., and Spzzichino, A., "Coded Aperture Imaging in X - and Gamma Ray Astronomy," Kluwer Academic Publishers 45, 349-403 (1987).

[27] DeWeert, M. J. and Farm, B. P., "Lensless coded aperture imaging with separable doubly Toeplitz masks," Proceedings of SPIE - The International Society for Optical Engineering 9109, 91090Q (2014).

[28] Rasband, W., "ImageJ." Available from: http://imagej.nih.gov/ij/. (Accessed 23 February 2016) (2011).

\footnotetext{
Content includes material subject to (C) Crown copyright (2017), Dstl. This material is licensed under the terms of the Open Government Licence except where otherwise stated. To view this licence, visit http://www.nationalarchives.gov.uk/doc/open-government-licence/version/3 or write to the Information Policy Team, The National Archives, Kew, London TW9 4DU, or email: psi@nationalarchives.gsi.gov.uk.
} 